\title{
New Clinically Feasible 3T MRI Protocol to Discriminate Internal Brain Stem Anatomy
}

\author{
(D) M.J. Hoch, (D) S. Chung, (D) N. Ben-Eliezer, (D).T. Bruno, (D) G.M. Fatterpekar, and (D).M. Shepherd
} $\stackrel{2}{2}=\Delta, D$

\begin{abstract}
SUMMARY: Two new 3T MR imaging contrast methods, track density imaging and echo modulation curve T2 mapping, were combined with simultaneous multisection acquisition to reveal exquisite anatomic detail at 7 canonical levels of the brain stem. Compared with conventional MR imaging contrasts, many individual brain stem tracts and nuclear groups were directly visualized for the first time at 3T. This new approach is clinically practical and feasible (total scan time $=20$ minutes), allowing better brain stem anatomic localization and characterization.
\end{abstract}

ABBREVIATIONS: $\mathrm{DEC}=$ direction-encoded color; $\mathrm{EMC}=$ echo modulation curve; $\mathrm{MLF}=$ medial longitudinal fasciculus; $\mathrm{PD}=$ proton-density; stDEC-TDI = short-track direction-encoded color track density imaging; stTDI = short-track track density imaging; TDI = track density imaging

$\mathbf{T}$ he brain stem contains many small but essential nuclei and fiber tracts that play key roles in motor, sensory, and autonomic regulation. ${ }^{1}$ Due to the compact, complex, and nonredundant anatomy of the brain stem, even small pathologic changes or lesions can have substantial clinical consequences. In particular, specific brain stem structures are affected in different subcortical neurodegenerative diseases such as Parkinson disease, multisystem atrophy, and progressive supranuclear palsy. ${ }^{2-5}$ Unfortunately, routine MR imaging by using thin-cut T1- and T2weighted images cannot resolve these individual small brain stem structures or measure subtle quantitative MR imaging tissue property changes that may escape visual detection by a

Received September 10, 2015; accepted after revision December 4.

From the Department of Radiology (M.J.H., S.C., N.B.-.E., M.T.B., G.M.F., T.M.S.) New York University Langone School of Medicine, New York, New York; and Center for Advanced Imaging Innovation and Research (S.C., N.B.-.E., T.M.S.), New York, New York.

Timothy Shepherd received research support from the National Institute of Aging (National Institutes of Health 1K23 AG048622-01). This research was supported, in part, by the Center for Advanced Imaging Innovation and Research, a National Institutes of Health National Institute of Biomedical Imaging and Bioengineering Biomedical Technology Resource Center (grant P41EB017183).

Abstract previously presented at: American Society of Neuroradiology Annual Meeting and the Foundation of the ASNR Symposium, April 25-30, 2015; Chicago, Illinois.

Please address correspondence to Timothy Shepherd, MD, PhD, Department of Radiology, Room 230D, New York University Langone School of Medicine, 660 First Ave, New York, NY 10016; e-mail: timothy.shepherd@nyumc.org

- Indicates open access to non-subscribers at www.ajnr.org

Indicates article with supplemental on-line table.

Indicates article with supplemental on-line photos.

$D$ Indicates article with supplemental on-line video.

http://dx.doi.org/10.3174/ajnr.A4685 neuroradiologist in the prodromal or early clinical stages of neurodegeneration.

MR imaging of the postmortem human brain stem by using $>1$-hour scan times, motionless dissected small samples, and contrast agent doping can generate high-quality, high-spatial-resolution structural MR imaging aids to guide image interpretation of in vivo subjects, ${ }^{6,7}$ but the MR imaging properties of postmortem nervous tissue are altered. ${ }^{8,9}$ The spatial and contrast resolution of conventional MR imaging obtainable in living subjects, particularly patients, is much more limited. Instead, in vivo brain stem MR imaging anatomy is inferred on the basis of craniocaudal section position, a few internal anatomic landmarks, and known surface topography. 7T MR imaging and alternative contrasts have been explored and have advantages over conventional MR imaging acquisitions. ${ }^{10-16}$ However, the sparse clinical availability of ultra-high-field MR imaging and extended scan times of advanced sequences (some individually $>20$ minutes) are both limitations. Susceptibility-weighted imaging of the brain stem can be limited by skull base artifacts, particularly at high magnetic fields.

Track density imaging (TDI) is a novel MR imaging postprocessing technique based on high-angular-resolution diffusion acquisitions that generate super-resolution images derived by whole-brain probabilistic streamline tractography. ${ }^{17,18}$ Images of $500-\mu \mathrm{m}$ isotropic resolution can be created in which pixel intensity reflects the number of probabilistic streamlines traversing the voxel and color reflects diffusion streamline orientations similar to those of conventional diffusion tensor imaging. ${ }^{19}$ These contrasts have been validated by using histology in animal models. ${ }^{20,21}$ We have combined a track density approach with recently developed simultaneous multisection acquisition diffusion MR 
imaging $^{22}$ to obtain TDI maps of brain stem structures in living subjects with clinically feasible scan times at $3 \mathrm{~T}$. We also have applied a new robust method for quantitating $\mathrm{T} 2$ and proton density in brain tissue on the basis of multisection, multiecho, spinecho acquisitions and a novel echo modulation curve (EMC) software package. ${ }^{23-25}$ We demonstrate the advantages of combining these 2 techniques to generate detailed in vivo depiction of internal brain stem anatomy with clinically feasible acquisition times at 3T MR imaging.

\section{MATERIALS AND METHODS MR Imaging Acquisition}

Three healthy right-handed (mean Edinburgh Handedness Inventory score of 78.6) adult subjects (mean age, $31 \pm 1$ years; 2 women) underwent noncontrast MR imaging of the head by using a 20-channel head and neck coil on a 3T MR imaging scanner (Skyra; Siemens, Erlangen, Germany). For quantitative T2 mapping, the multiecho spin-echo sequence included 15 echoes at 10-ms intervals (TE, 10-150 ms; TR, 5100 ms; matrix, $384 \times 234$; integrated Parallel Acquisition Techniques 2; 2-mm section thickness; 2 averages) and required 6 minutes. The high-angular-resolution diffusion sequence (3-mm isotropic resolution; matrix, $80 \times 80 ; 2$-section acceleration; 256 diffusion directions with b-value $=2500 \mathrm{~s} / \mathrm{mm}^{2} ; 8$ images with $b=0$; TR/TE, 3816/98 ms; 50 sections; phase-encoding in the anteroposterior direction) required 14 minutes. For susceptibility-induced distortion correction, 1 image with $b=0$ was acquired with phase-encoding in the posteroanterior direction. Additional conventional MR imaging sequences for comparison purposes included axial 2-mm-thick dual-echo T2-weighted MR imaging, 1-mm isotropic MPRAGE, 5-mm-thick axial FLAIR, and SWI (approximately 20 minutes additional scan time). Total scan time was 40 minutes. The above protocol was repeated on 1 subject 7 weeks after the initial scan to assess repeatability of the MR imaging contrasts.

\section{Quantitative T2 Maps}

T2 and proton density (PD) maps of the brain stem were postprocessed from the multi-spin-echo datasets by using a recently developed EMC T2 fitting software package, which relies on accurate Bloch simulations to model the exact signal evolution in multi-spin-echo pulse sequences by using the exact radiofrequency pulse shapes and other experimental parameters. ${ }^{23,24}$ Simulations were repeated for a range of T2 values (1-1000 ms) and $\mathrm{B}_{1}{ }^{+}$inhomogeneity values $\left(\mathrm{B}_{1}{ }^{+}=50 \% \ldots 130 \%\right)$, producing a data base of EMCs, each associated with a unique $\left(\mathrm{B}_{1}{ }^{+}, \mathrm{T} 2\right)$ value pair. The T2 parametric map was generated by matching experimentally acquired multi-spin-echo data to the EMC data base via 12-norm minimization of the difference between experimental and precalculated EMCs. PD maps were subsequently calculated by back-projecting the first echo image to time $\mathrm{t}=0$ by using the calculated T2 map. The software package is customwritten in Matlab (MathWorks, Natick, Massachusetts) and $\mathrm{C}++$ and then is executed with Matlab scripts and a graphic user interface. Detailed information and the software package can be found at http://cai2r.net/resources/software/emc-based-t2-mapping-package. All figures were created with a consistent T2 dynamic range of 20-120 ms and a normalized PD dynamic range of
0.2-0.6 (no units) for optimal brain stem contrast across the 7 canonical levels.

\section{Track Density Imaging}

The diffusion-weighted images were first corrected for susceptibility-induced distortion by using a phase reversal distortion-correction method (Topup tool in FSL; http://fsl.fmrib.ox.ac.uk/fsl/ fslwiki/topup), ${ }^{26}$ which is a part of the FSL toolbox (FMRIB Software Library; http://www.fmrib.ox.ac.uk/fsl). ${ }^{27}$ To generate the track density maps, we performed whole-brain probabilistic fiber-tracking by randomly seeding 4,000,000 tracks throughout the brain by using the MRtrix software package (http://neuro. debian.net/pkgs/mrtrix.html). ${ }^{28}$ The constrained spherical deconvolution technique ${ }^{29}$ with probabilistic streamlines was used to model multiple fiber orientations. The relevant fiber-tracking parameters were the following: $0.3-\mathrm{mm}$ step size, $9^{\circ}$ maximum angle between steps, $2.0-\mathrm{cm}$ maximum and $1.5-\mathrm{cm}$ minimum track length, and maximum harmonic order $\operatorname{lmax}=8$. Superresolution TDI and direction-encoded color (DEC) TDI maps were generated with a $500-\mu \mathrm{m}$ isotropic resolution. A "shorttrack" method was used for both TDI and DEC-TDI (now referred to as stTDI and stDEC-TDI) in which the maximum-tominimum track length ratio approached $1 .^{21}$ We used the conventional color scheme: blue (inferior-superior), green (anteroposterior), and red (left-right). In the case of stDEC-TDI, the color in each voxel can be determined by averaging the colors of all the streamline segments contained within the voxel. ${ }^{17}$

\section{MR Imaging Data Anatomic Analysis}

Postacquisition processing time for the quantitative T2 and track density maps was 15 and 180 minutes, respectively, by using an independent workstation. All MRIs were obtained with axial sections parallel to the anterior/posterior commissure line. For each subject, we then characterized anatomic detail in axial MR imaging sections from the conventional MR imaging protocol, quantitative T2, and short-track TDI parameter maps at 7 canonical anatomic levels of the brain stem ( similar to Hirsch et $\mathrm{al}^{30}$ ): rostral and caudal midbrain; rostral, middle, and caudal pons; and rostral and caudal medulla (Fig 1).The MR images were labeled according to the standard anatomic text of Duvernoy's Atlas of the Human Brain Stem and Cerebellum ${ }^{31}$ by consensus among 3 boardcertified neuroradiologists. Schematics were derived from Warner's Atlas of Neuroanatomy: With Systems Organization and Case Correlations. ${ }^{32}$ All figures were labeled with white matter tracts on the radiologic left and nuclei on the right of the image unless the structures were in a central location. A paired structure was only labeled unilaterally to avoid cluttering the image. Anatomic detail in the axial sections was compared across the 3 subjects and between different scan sessions for the single subject with repeat imaging.

\section{RESULTS}

Coregistered axial images of the brain stem at 7 discrete anatomic levels are shown in Figs 2-7 and On-line Figs 1 and 2 with labeled substructures (see the On-line Table for the complete list of labeled substructures, which are indicated in parentheses below). While brain stem anatomy is typically characterized in the axial plane, Fig 1 also demonstrates stDEC-TDI in a selected parasagittal section orientation for depicting axial section positions. Fig- 
ure 7 demonstrates reproducibility of brain stem anatomic assignments for 3 subjects, and Fig 8 demonstrates reproducibility for the same subject on different days. Movies of the various MR imaging parameter maps are provided on-line for the interested reader (Online Videos). A detailed, exhaustive discussion of the wealth of data available for each subject is beyond the scope of this current report. Below is a succinct discussion of the craniocaudal evolution of stTDI, stDEC-TDI, T2, and PD maps highlighting key findings.

The pyramidal tracts (16) were separable from the frontopontine (22) and occipitotemporal and parietopontine fibers (23) at the rostral midbrain on EMC PD maps (Fig 2E). The stDEC-TDI map (Fig $2 G$ ) shows the oculomotor nuclear complex (33) in green and the medial longitudinal fasciculus (MLF) (34) in light blue,

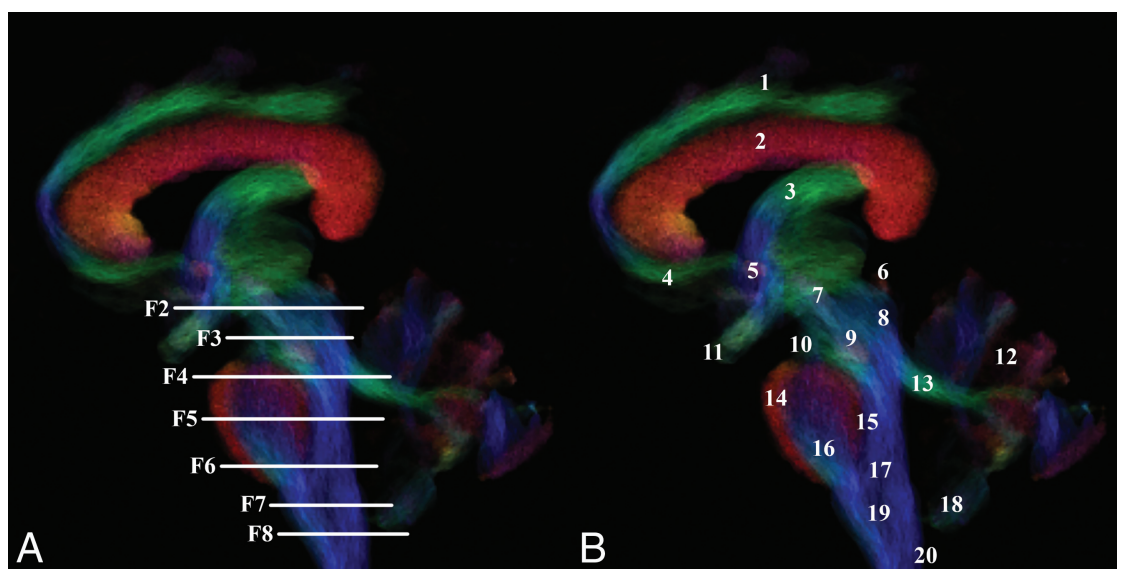

FIG 1. Parasagittal short-track direction-encoded color track density image of the brain stem. $A$, The 7 canonical axial anatomic levels of the brain stem are depicted with the section planes for Figs 2-6 and On-line Figs 1 and 2. B, Selected brain stem substructures are labeled to illustrate that TDI can also be used to make anatomic assignments in all 3 planes. Please refer to the On-line Table throughout this report for a complete list of labeled substructures for all figures. whereas the DEC-DTI map in this region shows a uniform green (Fig $2 C)$. The decussation of the superior cerebellar peduncles (9) at the level of the caudal midbrain is well-demarcated on EMC T2 maps (Fig 3F). The conventional T2-weighted image fails to distinguish the decussation (Fig $3 B$ ).

The high-intensity MLF (34) is clearly identified centrally on rostral pons stTDI maps (Fig 4H). On EMC maps, the pyramidal tracts (16) lose their conspicuity at the pons levels from intertwined traversing pontocerebellar fibers (14) (Fig 4E, -F). Within the midpons, the EMC PD image (On-line Fig $1 E$ ) better depicts the motor nucleus of the trigeminal nerve (46), and the medial lemnisci (15) and spinothalamic tract (26) are more conspicuous on the EMC T2 image (On-line Fig $1 F$ ) compared with their respective conventional images (On-line Fig $1 A,-B$ ). The stTDI maps show the facial nerve fascicles (50) from the colliculus and exiting the caudal pons (On-line Fig $2 H$ ).

The rostral or open medulla shows that the medial lemnisci (15) are easily distinguished from the pyramidal tract (16) on EMC T2 (Fig 5F). Dorsal plate nuclei of the hypoglossal (55) and the dorsal motor nucleus of the vagus nerve (56) are noted on stDEC-TDI maps (Fig $5 G$ ) dorsal to the MLF (34). Neither the conventional T2-weighted (Fig 5B) nor the DEC-DTI maps (Fig 5C) can directly demonstrate these structures. There is an intensity increase of the descending pyramidal tracts (16) on stTDI maps (Fig $6 H$ ) just superior to their medullary decussation.

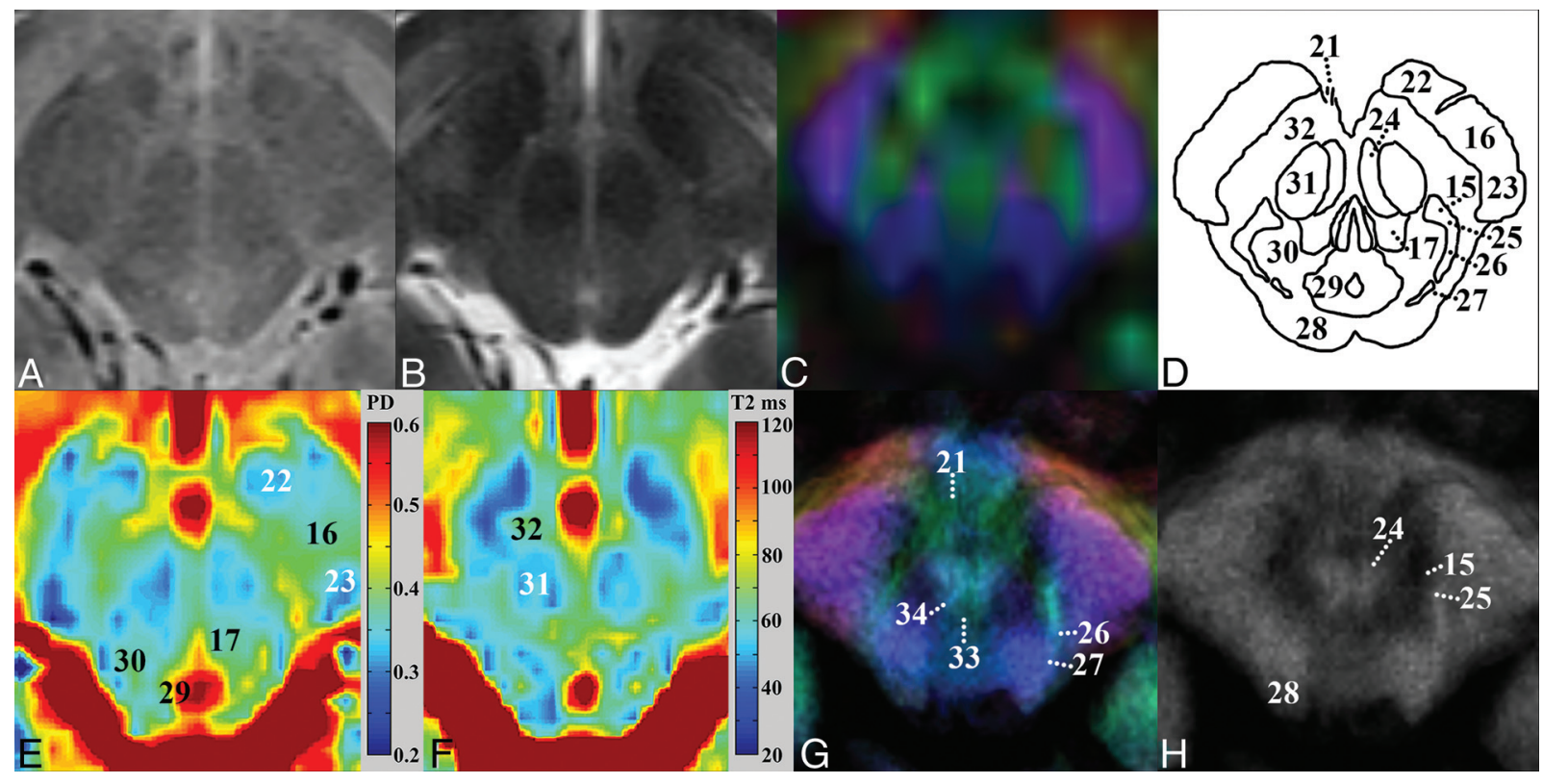

FIG 2. Rostral midbrain. Upper row: $A$, Proton-density weighted. $B$, T2-weighted. C, DEC-DTI. D, Anatomic schematic. Bottom row: E, EMC PD map. F, EMC T2 map. G, stDEC-TDI. H, stTDI. The lower row novel contrast images better depict brain stem anatomy compared with the upper row conventional images. Notice the appreciable difference in the EMC T2 value between the red nuclei (31) and the substantia nigra (32) compared with that of the standard multi-spin-echo T2 image. The stDEC-TDI map shows the oculomotor nuclear complex (33) in green and the MLF (34) in light blue, whereas the DEC-DTI map in this region shows a uniform green. 


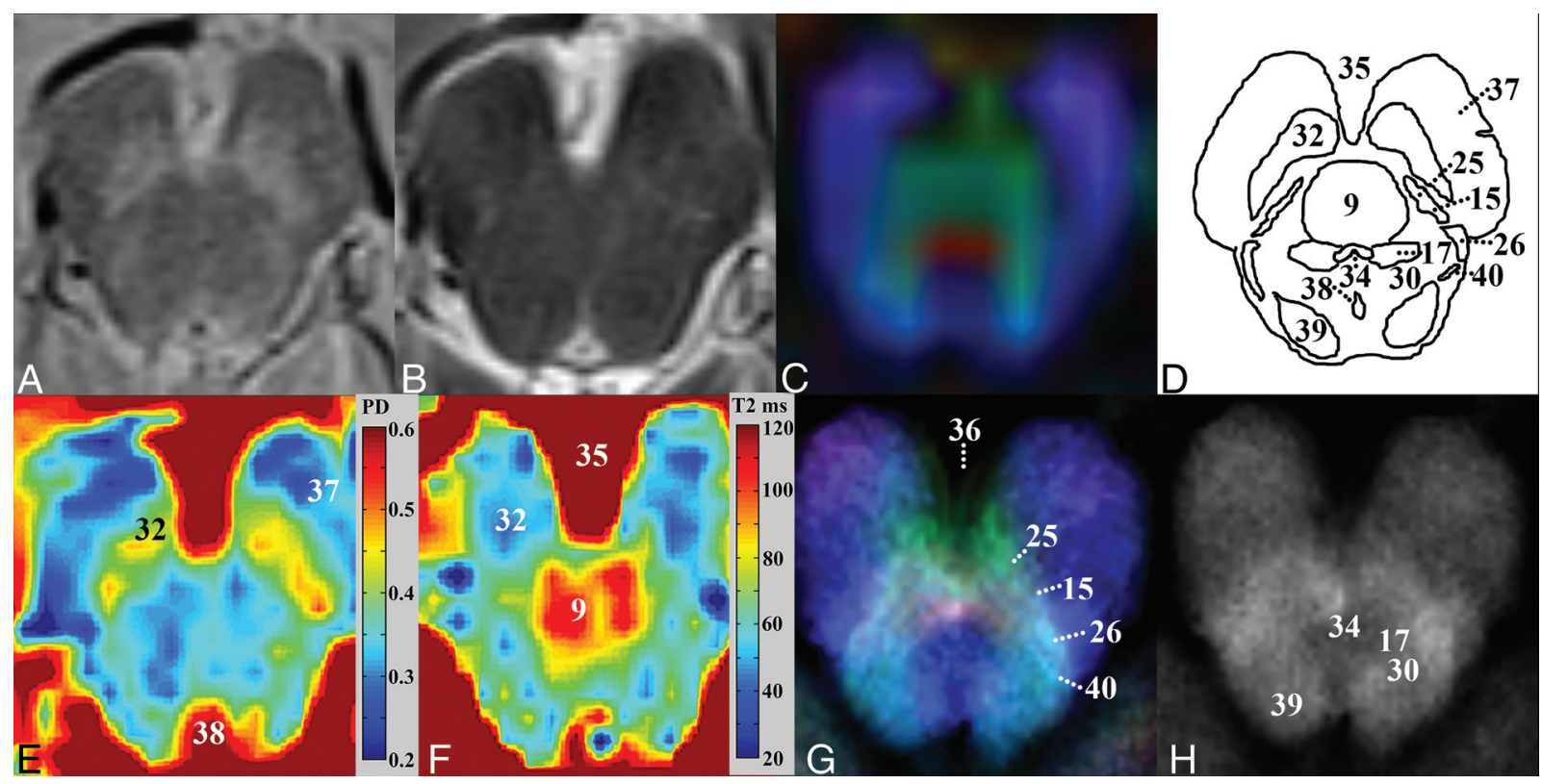

FIG 3. Caudal midbrain. Upper row: A, Proton-density weighted. B, T2-weighted. C, DEC-DTI. D, Anatomic schematic. Lower row: E, EMC PD map. F, EMC T2 map. G, stDEC-TDI. H, stTDI. The ventral trigeminothalamic tract (25), medial lemniscus (15), spinothalamic tract (26), and lateral lemniscus (40) are better outlined on stDEC-TDI compared with conventional DEC-DTI. The EMC T2 map clearly shows the decussation of the superior cerebellar peduncle (9) as an intense red structure. The conventional T2-weighted image fails to distinguish the decussation from adjacent structures.

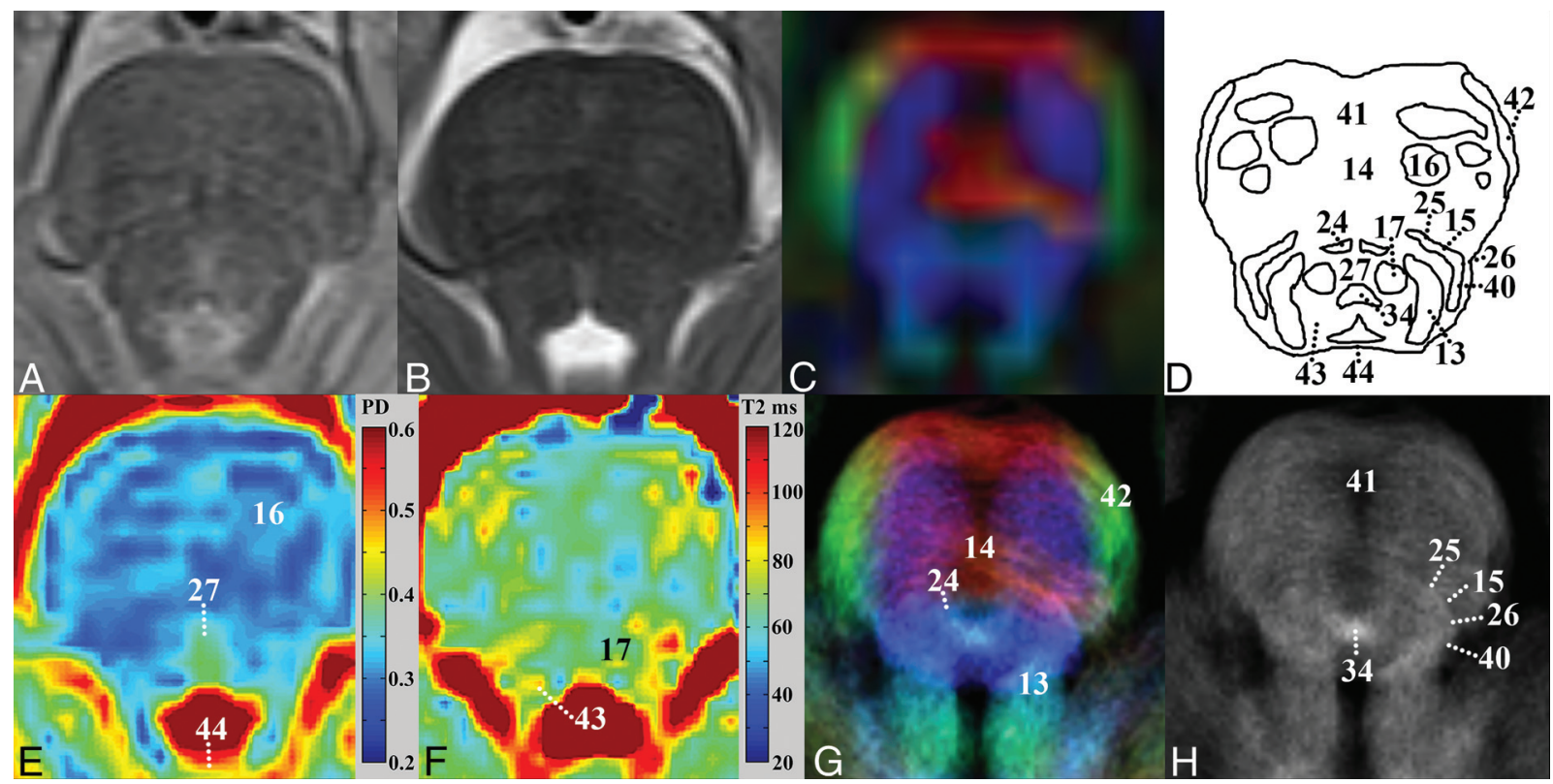

FIG 4. Rostral pons. Upper row: $A$, Proton-density weighted. $B$, T2-weighted. C, DEC-DTI. D, Anatomic schematic. Lower row: E, EMC PD map. F, EMC T2 map. G, stDEC-TDI. H, stTDI. The MLF (34) qualitatively has the highest signal intensity on stTDI and stDEC-TDI, suggesting that many probabilistic streamlines cross through these imaging voxels. The DEC-DTI map has uniform intensity, and the MLF cannot be appreciated. The locus coeruleus (43) can now be seen medial to the superior cerebellar peduncles (13) on EMC T2 and PD maps.

EMC PD maps appeared consistently superior to T2 maps for identifying nuclear groups, for example the trigeminal motor nuclei (46) on On-line Fig 1E, -F. Overall, 15 groups of bilateral paired nuclei $(19,28,31,32,33,39,43,45,46,51,55,56,57,63$, 64) were identified on PD maps as opposed to T2 maps where only 9 nuclear pairs $(19,28,31,32,39,43,46,55,56)$ were identified. Both stTDI and stDEC-TDI identified 11 pairs of nuclei $(19,28,31,32,33,39,51,55,56,63,64)$, especially those with identifiable fascicles (eg, oculomotor [33] and trigeminal nuclei
[51]). Additional small nuclei were not distinct on any of the new MR imaging parameter maps (including the abducens, ambiguus, trochlear, cochlear, salivatory, and superior olives).

Aside from structures that can be recognized on conventional MR imaging from their characteristic surface topography, our protocol revealed many deeper substructures. The spinal tract of cranial nerve V (58) was seen on stTDI and stDEC-TDI maps in the dorsolateral midpons extending to the caudal medulla (Figs 5-6 and On-line Figs 1 and $2 G,-H)$. Descending small central 


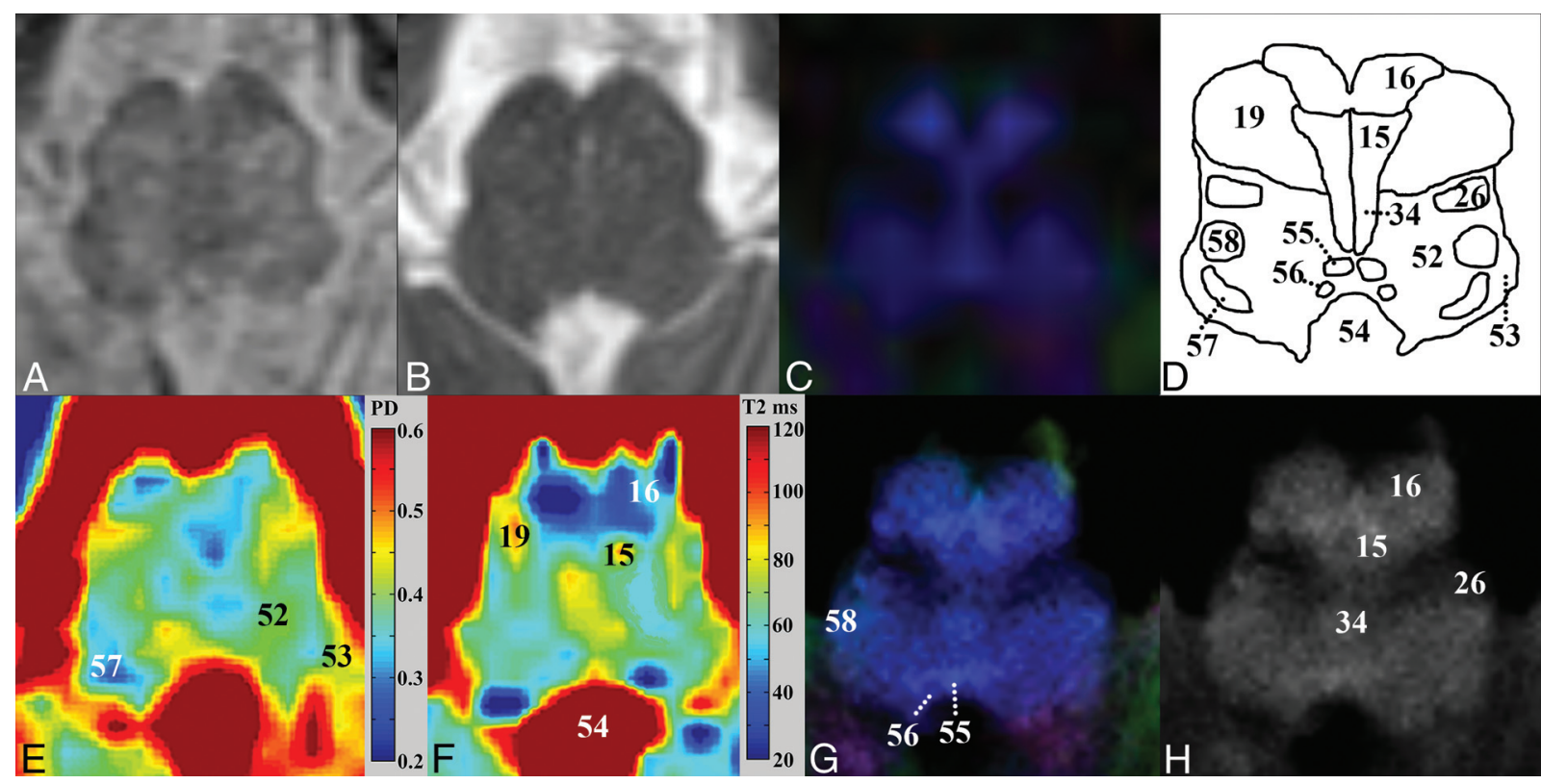

FIG 5. Rostral medulla. Upper row: A, Proton-density weighted. B, T2-weighted. C, DEC-DTI. D, Anatomic schematic. Lower row: E, EMC PD map. F, EMC T2 map. G, stDEC-TDI. H, stTDI. More brain stem substructures are directly identified with our protocol. For example, the medial lemnisci (15) are easily distinguished from the pyramidal tracts (16) on EMC T2. Dorsal plate nuclei of the hypoglossal (55) and the dorsal motor nucleus of the vagus nerve (56) are noted on both stTDI maps. Neither the conventional T2-weighted nor the DEC-DTI map can directly demonstrate these structures.

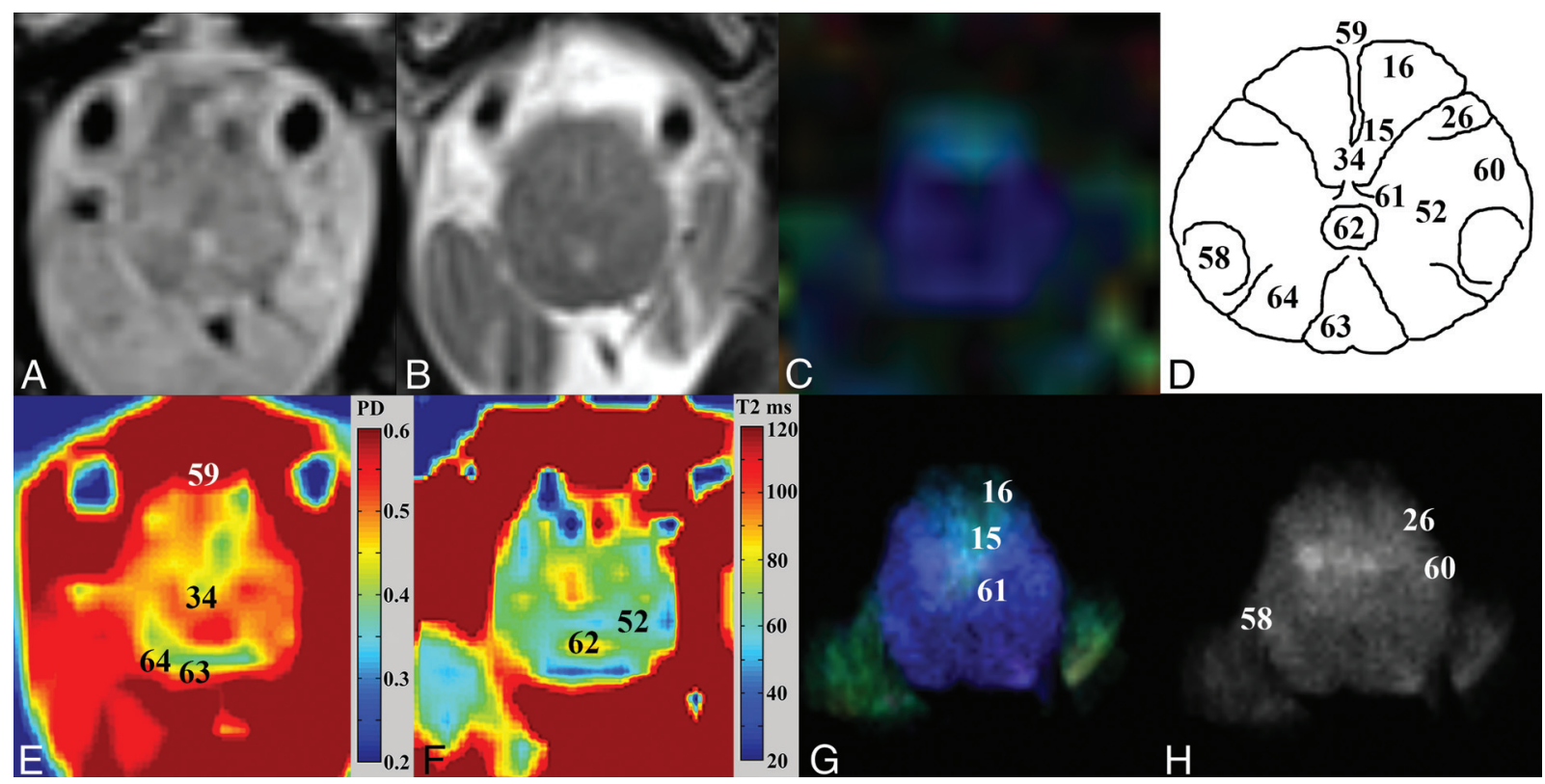

FIG 6. Caudal medulla. Upper row: A, Proton-density weighted. B, T2-weighted. C, DEC-DTI. D, Anatomic schematic. Lower row: E, EMC PD map. F, EMC T2 map. G, stDEC-TDI. H, stTDI. There is a qualitative difference in the T2, PD, and stTDI values between the right and left pyramidal tracts (16) above the decussation, which may reflect right-handedness in this subject (best seen in $E$ ). This left-versus-right signal difference is not appreciable on the routine T2- or PD-weighted images or the DEC-DTI. In addition, stDEC-TDI can show the subtle red streamline internal arcuate (61) crossing the midline, another advantage over DEC-DTI.

tracts that synapse with the spinal cord, the central tegmental tract (17) and reticular formation $(30,48,52)$, were not seen in their entirety because they are difficult to distinguish from each other. However, on stTDI, the rubrospinal tracts were seen medial to the red nuclei (31) in the rostral midbrain (Fig 2H). The tectospinal tract (27) could be seen at the rostral midbrain and rostral pons levels (Figs $2 G$ and $4 E$ ). Furthermore, stTDI clearly was superior to conventional DTI parameter maps derived of these sample data-that is, in the caudal midbrain, the ventral trigeminothalamic tract (25), spinothalamic tract (26), and lateral lemniscus (40) were obvious on stTDI and stDEC-TDI, but not appreciated on DEC-DTI (Fig 3C).

\section{DISCUSSION}

Our new multicontrast protocol with clinically feasible acquisition times gains spatial resolution and anatomic contrast by using 


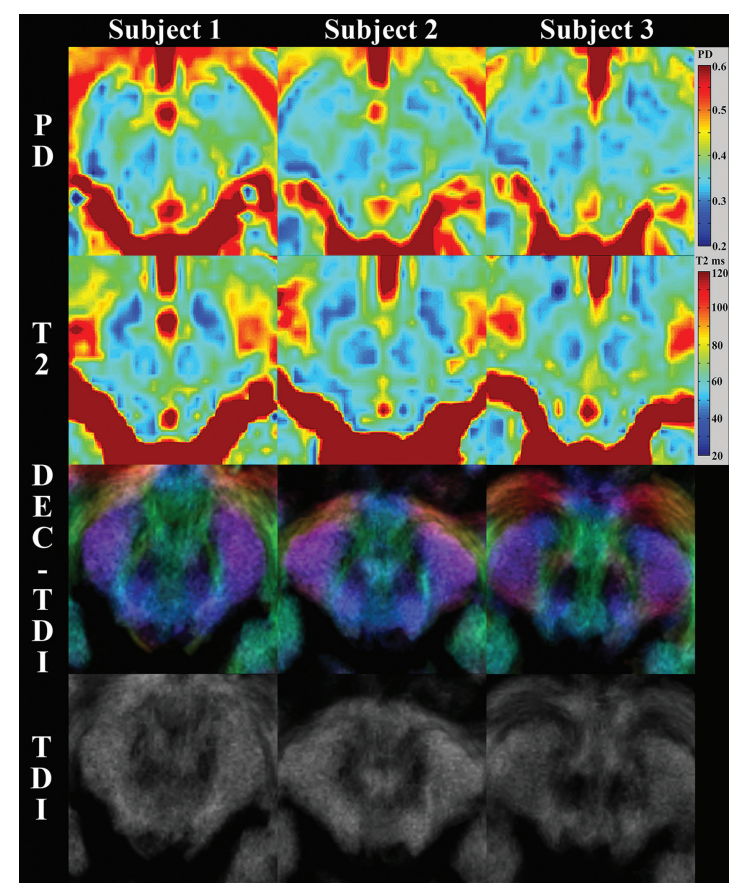

FIG 7. EMC PD and T2, stDEC-TDI, and stTDI maps at the rostral midbrain for 3 right-handed subjects (mean age, $31 \pm 1$ years; 2 women; mean Edinburgh Handedness Inventory score, 78.6) demonstrate excellent reproducibility for the novel MR imaging acquisition and postprocessing protocol among healthy controls. Detailed anatomy for the different MR imaging contrast at this axial level is depicted in Fig 2. There are subtle differences in the subthalamic anatomy ventral and superior to the midbrain (note subject 2 in the stDEC-TDI and stTDI images) attributed to slight angulation differences in the placement of the imaging plane parallel to the anterior/posterior commissure line.

postprocessing methods to reveal structures beyond the grasp of conventional MR imaging. This protocol has the potential to become widely applicable to many brain stem pathologies and generate new specific imaging biomarkers. Furthermore, in vivo visualization of these small structures does not have to be limited to large academic centers with ultra-high-field MR imaging or opportunities for extended-length scan protocols. The only caveat is that radiologists may need to relearn detailed brain stem anatomy not typically required when reading conventional MR imaging.

A goal is clinical implementation, and a whole-brain diffusion sequence was chosen because patients may have diffusion abnormalities outside the brain stem. To decrease our scan time, we applied a simultaneous multisection acquisition to acquire the data. ${ }^{22}$ Whole-brain coverage is not always necessary, and a reduced z-FOV covering only the brain stem may be an alternative option in research subjects. TDI needs a sufficient number of DWI directions and SNR to reduce uncertainty in estimating white matter fiber tracts. TDI benefits from high-angular-resolution diffusion imaging and higher b-values to resolve areas of "crossing fibers." ${ }^{33}$ It is essential to use an MR imaging model that accounts for the presence of multiple fiber directions within a voxel; therefore, the constrained spherical deconvolution method and probabilistic streamlines are used. ${ }^{29}$ The gain in actual resolution beyond the authentic imaging voxel differs from image interpolation that does not provide any extra information.

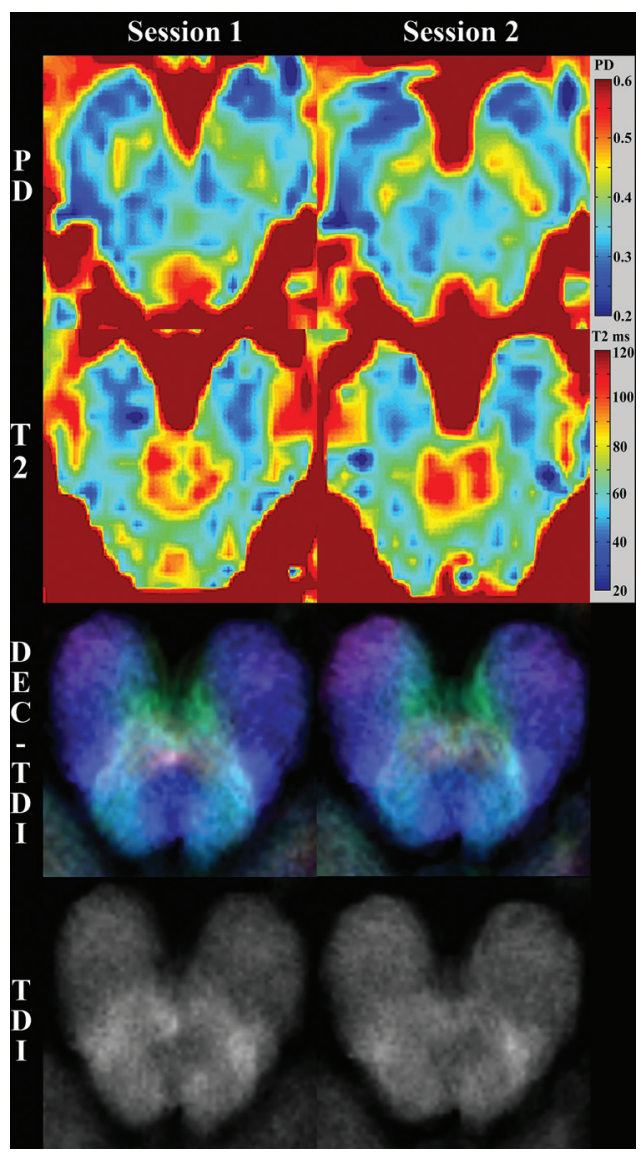

FIG 8. Single axial section at the caudal midbrain with EMC and stTDI maps repeated in the same subject 7 weeks apart demonstrating consistent contrasts within the subject. Detailed anatomy for the different MR imaging contrast at this axial level is depicted in Fig 3.

Nervous tissue water diffusion behavior is field-independent but technically challenging to characterize at $7 \mathrm{~T}$. Deistung et al ${ }^{16}$ used TDI to successfully parcellate individual brain stem substructures at 7T. Our TDI maps show more structures than samesource DTI color maps and the TDI maps of Deistung et al because we used a short-track TDI scaling method. Short brain stem fiber tracks have fewer streamlines due to the random seed point placement technique, which leads to a reduced intensity of the generated tracts in whole-brain TDI maps. ${ }^{18}$ With a short-track method and increasing the number of seed points, this effect is alleviated and a reasonable contrast-to-noise ratio is sustained. ${ }^{18,21}$ Spherical-deconvolution informed filtering is a recently introduced alternative scaling method, which could be useful in future anatomic studies. ${ }^{34}$

TDI has the potential to quantitatively characterize healthy and diseased local structure connectivity ${ }^{35}$ if acquisition parameters are consistent. ${ }^{29}$ Ziegler et al ${ }^{36}$ showed nigrostriatal changes in Parkinson disease. Currently, there is debate on the validity of quantification due to tractography biases and errors and which method should be used. Average pathlength mapping was first introduced ${ }^{37}$ and has now been followed by the more direct apparent fiber density. ${ }^{38}$ The source DWI resolution (3 mm isotropic) is relatively coarse and could potentially cause quantification errors in our maps.

EMC T2 mapping models both stimulated echoes and radio- 
frequency inhomogeneities inherent to clinical 3T imaging in real patients. This feature allows the quantification of T2 and PD values in a manner independent of the scanner and parameter values and at clinically feasible time scales. ${ }^{25}$ These maps can be used to increase anatomic contrast by emphasizing subtle tissue property differences over a dynamic range that remains compressed on standard T2-weighted images.

SWI delineates some in vivo brain stem anatomy well; however, the best results have required 7T MR imaging. Eapen et $\mathrm{al}^{14}$ focused on the human midbrain dopaminergic system. Gizewski et $\mathrm{al}^{10}$ and Deistung et $\mathrm{al}^{16}$ also used SWI in their protocols at 7T to parcellate individual brain stem substructures. However, in addition to increased aliasing and susceptibility artifacts at 7T, such high-field systems are not widely available or currently approved for clinical use in the United States. Instead of SWI, our protocol focuses on robust T2 characterization at clinically available 3T, in which there is less field-dependent T2 shortening compared with $7 \mathrm{~T}^{39}$

A limitation of using fast T2 mapping protocols, similar to routine spin-echo imaging, is pulsation artifacts from nearby blood vessels. The vertebral and basilar arteries and the sigmoid sinuses can cause artifactual low T2 and PD values within the lower brain stem. In initial studies, we realized that increasing the bandwidth from 199 to $600 \mathrm{~Hz} /$ pixel helped reduce these pulsation artifacts.

The protocol makes direct identification of more structures possible, with additional structures identified by the positions relative to other structures. Before clinical implementation, anatomic assignments in correlation with reconstructed T2 and TDI images have to be verified with larger postmortem studies. Our method is by no means perfection but is an improvement in the ratio of directly versus indirectly visualized structures at 3T. A full assessment of the range of quantitative values for these new MR imaging parameter maps of the brain stem is beyond the scope of this initial report but will be the subject of ongoing follow-up research in a larger cohort of subjects that also assesses differences in age, sex, and handedness.

\section{CONCLUSIONS}

Clinically feasible MR imaging at 3T with novel TDI and T2 mapping better characterizes the detailed anatomy of the in vivo human brain stem compared with routine clinical sequences. Increased contrast EMC PD maps excelled at identifying nuclear groups. Super-resolution TDI maps showed several small fiber tracts not previously seen at 3T. Each contrast map has its own advantages, but for best results, use of multiple independent maps provides the optimal approach to identify different anatomic substructures. We are optimistic that this protocol has the potential to identify future imaging biomarkers for a wide assortment of clinical pathologies in the brain stem.

Disclosures: Noam Ben-Eliezer-RELATED: Grant: National Institutes of Health (P41 EB017183)*; UNRELATED: Patents (planned, pending or issued): New York University School of Medicine, ${ }^{*}$ Comments: Ben-Eliezer N, Block KT, "Method and Device For Accurate Quantification of T2 Relaxation Times based on Fast Spin-Echo NMR Sequences," 2013 (provisional patent application number 61/767,663). Timothy M. Shepherd-RELATED: Grant: National Institute of Aging (1K23 AG048622-01)*; UNRELATED: Patents (planned, pending or issued): I have 3 provisional or pending patents, devices for image-guided procedures that are unrelated to this work. Neither I nor my institution has received royalties or license fees for these inventions at this time; Other: I spoke for Siemens at both the International Society for Magnetic Resonance in Medicine and Human Brain Mapping 2015 meetings but did not accept any fees, honorarium, or reimbursement for travel. *Money paid to the institution.

\section{REFERENCES}

1. Carpenter MB, Strong OS, Truex RC. Human Neuroanatomy: (Formerly Strong and Elwyn's Human Neuroanatomy). 7th ed. Baltimore: Lippincott Williams \& Wilkins; 1976

2. Urbaìn P. Brainstem Disorders. Berlin: Springer-Verlag; 2011

3. Janzen J, van 't Ent D, Lemstra AW. The pedunculopontine nucleus is related to visual hallucinations in Parkinson's disease: preliminary results of a voxel-based morphometry study. J Neurol 2012; 259:147-54 CrossRef Medline

4. Rolland Y, Verin M, Payan CA, et al; NNIPPS Study Group. A new MRI rating scale for progressive supranuclear palsy and multiple system atrophy: validity and reliability. J Neurol Neurosurg Psychiatry 2011;82:1025-32 CrossRef Medline

5. Makino T, Ito S, Kuwabara S. Involvement of pontine transverse and longitudinal fibers in multiple system atrophy: a tractographybased study. J Neurol Sci 2011;303:61-66 CrossRef Medline

6. Solsberg MD, Fournier D, Potts DG. MR imaging of the excised human brainstem: a correlative neuroanatomic study. AJNR Am J Neuroradiol 1990;11:1003-13 Medline

7. Soria G, De Notaris M, Tudela R, et al. Improved assessment of ex vivo brainstem neuroanatomy with high-resolution MRI and DTI at 7 Tesla. Anat Rec (Hoboken) 2011;294:1035-44 CrossRef Medline

8. Shepherd TM, Flint JJ, Thelwall PE, et al. Postmortem interval alters the water relaxation and diffusion properties of rat nervous tissue: implications for MRI studies of human autopsy samples. Neuroimage 2009;44:820-26 CrossRef Medline

9. Shepherd TM, Thelwall PE, Stanisz GJ, et al. Aldehyde fixative solutions alter the water relaxation and diffusion properties of nervous tissue. Magn Reson Med 2009;62:26-34 CrossRef Medline

10. Gizewski ER, Maderwald S, Linn J, et al. High-resolution anatomy of the human brain stem using 7-T MRI: improved detection of inner structures and nerves? Neuroradiology 2014;56:177-86 CrossRef Medline

11. Naganawa S, Yamazaki M, Kawai H, et al. Anatomical details of the brainstem and cranial nerves visualized by high resolution readout-segmented multi-shot echo-planar diffusion-weighted images using unidirectional MPG at 3 T. Magn Reson Med Sci 2011;10: 269-75 CrossRef Medline

12. Nagae-Poetscher LM, Jiang H, Wakana S, et al. High-resolution diffusion tensor imaging of the brain stem at $3 \mathrm{~T}$. AJNR Am JNeuroradiol 2004;25:1325-30 Medline

13. Salamon N, Sicotte N, Alger J, et al. Analysis of the brain-stem white matter tracts with diffusion tensor imaging. Neuroradiology 2005; 47:895-902 CrossRef Medline

14. Eapen M, Zald DH, Gatenby JC, et al. Using high-resolution MR imaging at 7T to evaluate the anatomy of the midbrain dopaminergic system. AJNR Am J Neuroradiol 2011;32:688-94 CrossRef Medline

15. Cho ZH. 7.0 Tesla MRI Brain White Matter Atlas. 2nd ed. Berlin: Springer-Verlag; 2015

16. Deistung A, Schäfer A, Schweser F, et al. High resolution MR imaging of the human brainstem in vivo at 7 Tesla. Front Hum Neurosci 2013; 7:710 CrossRef Medline

17. Calamante F, Tourneir JD, Jackson GD, et al. Track density imaging (TDI): super-resolution white matter imaging using whole-brain track density mapping. Neuroimage 2010;53:1233-43 CrossRef Medline

18. Calamante F, Tournier JD, Heidemann RM, et al. Track density imaging (TDI): validation of super resolution property. Neuroimage 2011;56:1259-66 CrossRef Medline

19. Pajevic S, Pierpaoli C. Color schemes to represent the orientation of anisotropic tissues from diffusion tensor data: application to white 
matter fiber tract mapping in the human brain. Magn Reson Med 2000:43:921 Medline

20. Ullmann JF, Calamante F, Collin SP, et al. Enhanced characterization of the zebrafish brain as revealed by super-resolution trackdensity imaging. Brain Struct Funct 2015;220:457-68 CrossRef Medline

21. Calamante F, Tournier JD, Kurniawan ND, et al. Super-resolution track-density imaging studies of mouse brain: comparison to histology. Neuroimage 2012;59:286-96 CrossRef Medline

22. Setsompop K, Gagoski BA, Polimeni JR, et al. Blipped-controlled aliasing in parallel imaging for simultaneous multislice echo planar imaging with reduced g-factor penalty. Magn Reson Med 2012;67: 1210-24 CrossRef Medline

23. Ben-Eliezer N, Sodickson DK, Block KT. Rapid and accurate T2 mapping from multi-spin-echo data using Bloch-simulation-based reconstruction. Magn Reson Med 2015;73:809-17 CrossRef Medline

24. Ben-Eliezer N, Feng L, Block KT, et al. Accelerated in vivo mapping of $\mathrm{T} 2$ relaxation from radially undersampled datasets using compressed sensing and model-based reconstruction. In: Proceeding of the Annual Meeting of International Society for Magnetic Resonance in Medicine, Milan, Italy. May 10-16, 2014

25. Ben-Eliezer N, Sodickson DK, Shepherd T, et al. Accelerated and motion-robust in vivo T2 mapping from radially undersampled data using Bloch-simulation-based iterative reconstruction. Magn Reson Med 2015 Apr 17. [Epub ahead of print] CrossRef Medline

26. Andersson JL, Skare S, Ashburner J. How to correct susceptibility distortions in spin-echo echo-planar images: application to diffusion tensor imaging. Neuroimage 2003;20:870-88 CrossRef Medline

27. Smith SM, Jenkinson M, Woolrich MW, et al. Advances in functional and structural MR image analysis and implementation as FSL. Neuroimage 2004;23(suppl 1):S208-19 CrossRef Medline

28. Tourneir JD, Calamante F, Connelly A. MRtrix: diffusion tractography in crossing fiber regions. Int J Imaging Syst Technol 2012;22: 53-66 CrossRef
29. Tournier JD, Calamante F, Connelly A. Robust determination of the fibre orientation distribution in diffusion MRI: non-negativity constrained super-resolved spherical deconvolution. Neuroimage 2007;35:1459-72 CrossRef Medline

30. Hirsch WL, Kemp SS, Martinez AJ, et al. Anatomy of the brainstem: correlation of in vitro MR images with histologic sections. AJNR Am J Neuroradiol 1989;10:923-28 Medline

31. Naidich TP, Duvernoy HM, Delman BD, et al. Duvernoy's Atlas of the Human Brain Stem and Cerebellum. Vienna: Springer-Verlag; 2008

32. Warner JJ. Atlas of Neuroanatomy: With Systems Organization and Case Correlations. Boston: Butterworth-Heinemann; 2001

33. Tuch DS, Reese TG, Wiegell MR, et al. High angular resolution diffusion imaging reveals intravoxel white matter fiber heterogeneity. Magn Reson Med 2002;48:577-82 CrossRef Medline

34. Smith RE, Tournier JD, Calamante F, et al. SIFT: spherical-deconvolution informed filtering of tractograms. Neuroimage 2013;67:298 312 CrossRef Medline

35. Willats L, Raffelt D, Smith RE, et al. Quantification of trackweighted imaging (TWI): characterisation of within-subject reproducibility and between-subject variability. Neuroimage 2014;87: 18-31 CrossRef Medline

36. Ziegler E, Rouillard M, André E, et al. Mapping track density changes in nigrostriatal and extranigral pathways in Parkinson's disease. Neuroimage 2014;99:498-508 CrossRef Medline

37. Calamante F, Tournier JD, Smith RE, et al. A generalised framework for super-resolution track-weighted imaging. Neuroimage 2012;59: 2494-503 CrossRef Medline

38. Calamante F, Smith RE, Tournier JD, et al. Quantification of voxelwise total fibre density: investigating the problems associated with track-count mapping. Neuroimage 2015;117:284-93 CrossRef Medline

39. Levitt MH. Spin Dynamics: Basics of Nuclear Magnetic Resonance. 2nd ed. Chichester: John Wiley \& Sons; 2008 